\title{
Impact of interplanetary shock on the ULF wave activity: A case study of the storm sudden commencement on September 22, 1999
}

\author{
J. Kangas ${ }^{1}$, J. Kultima ${ }^{1}$, A. Guglielmi ${ }^{2}$, A. Potapov ${ }^{3}$, and K. Hayashi ${ }^{4}$ \\ ${ }^{1}$ Sodankylä Geophysical Observatory, FIN-99600 Sodankylä, Finland \\ ${ }^{2}$ Institute of Physics of the Earth, 123810 Moscow, Russia \\ ${ }^{3}$ Institute of Solar-Terrestrial Physics, 664033 Irkutsk, Russia \\ ${ }^{4}$ Department of Earth and Planetary Science, University of Tokyo, Tokyo 113-0033, Japan
}

(Received January 15, 2001; Revised September 14, 2001; Accepted October 4, 2001)

\begin{abstract}
We have analyzed short-period geomagnetic pulsations measured by the north-south chain of pulsation magnetometers in Finland in association with the storm sudden commencement (SSC) recorded on September 22, 1999 at 12:22 UT. We observe that structured Pc 1 pulsations with amplitude modulation (modulation period is about 1000 seconds) were excited before the SSC whereas intense wave elements with rising frequency appear soon after SSC. It is concluded that the first effect is due to the interaction of the Earth's magnetosphere with the interplanetary foreshock region. The second one is likely the result of modification of Pc 1 waves due to interaction between the magnetosphere and the shock front structure leading to compression of the magnetosphere during the initial phase of the magnetic storm.
\end{abstract}

\section{Introduction}

Ultra low frequency (ULF) electromagnetic waves attract our attention as an interesting natural phenomenon which provides information on the physical processes in the space plasma (e.g., Guglielmi and Pokhotelov, 1996; Kangas et al., 1998). A separate class of ULF-emissions includes short-period oscillations related to sudden impulses in the geomagnetic field caused by interplanetary shock impact on the magnetosphere. A number of authors (Troitskaya, 1961; Tepley and Wentworth, 1962; Heacock and Hessler, 1965; Troitskaya and Guglielmi, 1967; Saito and Matsushita, 1967; Kokubun and Oguti, 1968; Olson and Lee, 1983; Kangas et al., 1986, 1998; Anderson and Hamilton, 1993) have studied Pc 1 pulsations excited by storm sudden commencements (SSC). Less common are Pc 1 emissions observed several hours before SSC (Troitskaya, 1964; Kangas et al., 1998; Guglielmi et al., 2000). Generation of the post-SSC Pc 1 is connected with magnetospheric compression due to its interaction with an interplanetary shock wave (Olson and Lee, 1983; Anderson and Hamilton, 1993; Kangas et al., 1998). Preshock Pc 1's are generated due to the magnetospheric interaction with upstream turbulence of an interplanetary shock wave (Kangas et al., 1998; Guglielmi et al., 2000). It is not clear yet, whether this process is limited only by the magnetospheric compression or involves some other mechanisms.

The present paper is motivated firstly by the S-RAMP September 1999 Space Weather Month campaign (Baker and Koskinen, 1999), and secondly by our special interest

Copy right (C) The Society of Geomagnetism and Earth, Planetary and Space Sciences (SGEPSS); The Seismological Society of Japan; The Volcanological Society of Japan; The Geodetic Society of Japan; The Japanese Society for Planetary Sciences. in the problem of impact of interplanetary blast shocks generated by the solar flares on the ULF wave activity in the Earth's magnetosphere (Kangas et al., 1986, 1998). In particular, we have established recently an elevation of Pc 1 wave activity prior to magnetic sudden impulse SI. We obtained this result by statistical analysis of the long-term observations of Pc 1, and we explain the effect as a specific response of the magnetosphere to the impact of the interplanetary foreshock (Guglielmi et al., 2000). The 1999 Space Weather Month campaign (Baker and Koskinen, 1999) gives us a possibility to support the statistical result by the analysis of an individual event. The main goal of this work is to follow the details of processes resulting in both pre- and postSSC short-period emissions by comparing Pc 1 observations at the net of magnetometers with simultaneous satellite measurements in the solar wind for the specific SSC - Pc 1 event on September 22, 1999.

The magnetic storm was triggered by the interplanetary shock which arrived at the Earth on September 22, 1999, and which manifests itself as the SSC at 12:22 UT. Before this time we observe a long interval of the Pc 1 wave activity which is evidently related to the shock arrival. This "foreshock part" of complex ULF wave emissions associated with the interplanetary shock is described in the Section 2 . The Pc 1 wave activity preceding the shock demonstrates an interesting peculiarity: it is modulated with a period of several minutes. The Section 3 is devoted to the "aftershock part" of the ULF event, namely to the intense wave patterns with rising frequency which started in the wake of the shock arrival. These elements remind an IPDP event (Intervals of Pulsations of Diminishing Period) but have some features that do not allow to consider them as typical ones (abnormal high upper frequency, atypical local time of the 
Table 1. Locations of the pulsation magnetometer stations.

\begin{tabular}{|c|c|c|c|c|c|}
\hline \multirow{3}{*}{ Station } & \multirow{2}{*}{\multicolumn{2}{|c|}{ Geographic coordinates }} & \multirow{2}{*}{\multicolumn{2}{|c|}{$\begin{array}{c}\text { Corrected geomagnetic } \\
\text { coordinates }\end{array}$}} & \multirow{3}{*}{$L$-value } \\
\hline & & & & & \\
\hline & latitude & longitude & latitude & longitude & \\
\hline Kilpisjärvi & 69.0 & 20.9 & 65.9 & 104.2 & 6.1 \\
\hline Ivalo & 68.6 & 27.3 & 65.1 & 108.9 & 5.7 \\
\hline Sodankylä & 67.4 & 26.4 & 64.0 & 107.4 & 5.3 \\
\hline Rovaniemi & 66.8 & 25.9 & 63.4 & 106.6 & 5.1 \\
\hline Oulu & 65.1 & 25.9 & 61.7 & 105.6 & 4.5 \\
\hline Nurmijärvi & 60.5 & 24.6 & 57.0 & 102.4 & 3.4 \\
\hline Borok & 58.0 & 39.0 & 53.9 & 114.3 & 2.9 \\
\hline Mondy & 51.6 & 100.8 & 46.7 & 173.6 & 2.1 \\
\hline
\end{tabular}
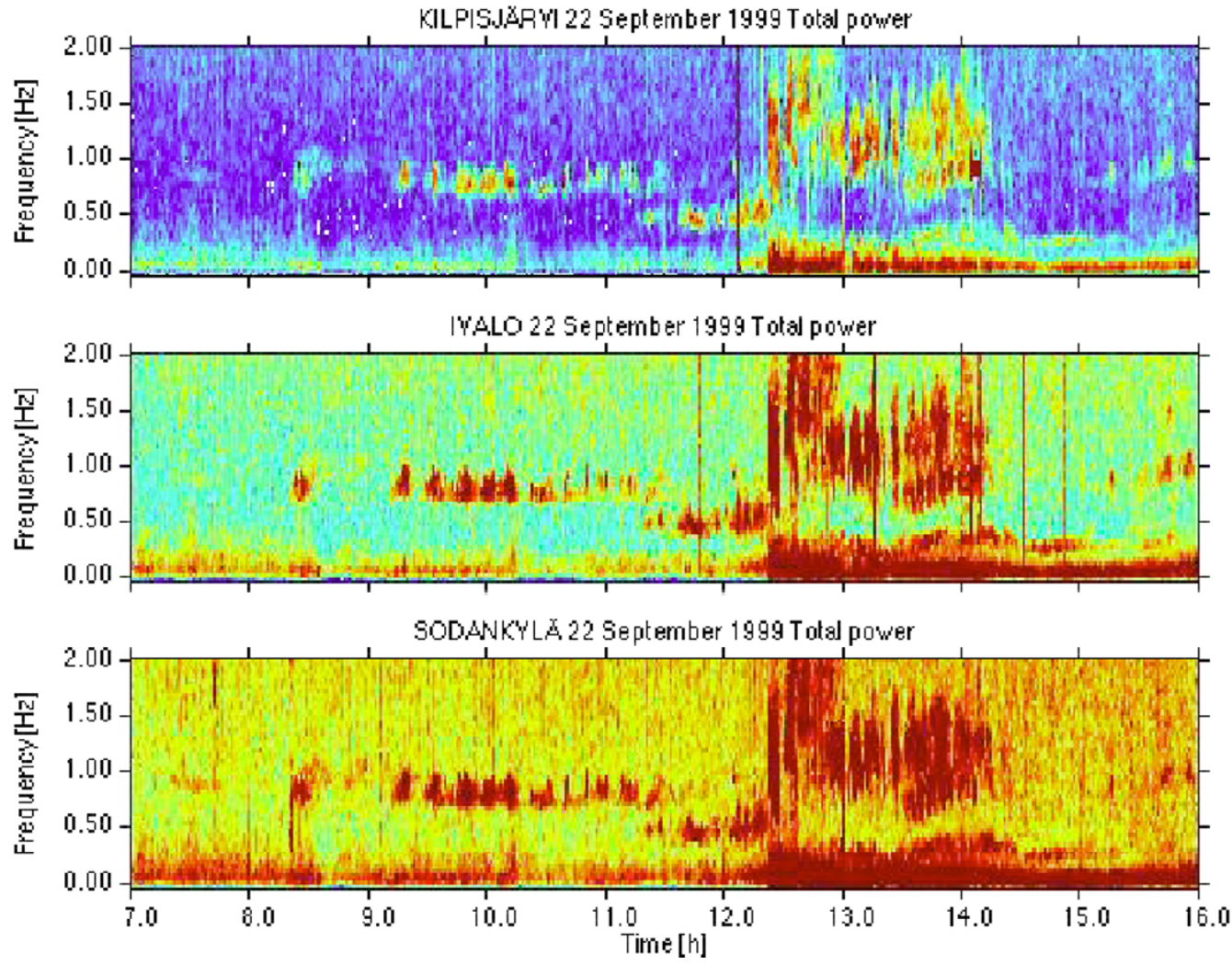

Fig. 1. Dynamical spectra of the short-period magnetic pulsations in Kilpisjärvi (KIL, $L=6.1$ ), Ivalo (IVA, $L=5.7)$, and Sodankylä (SOD, $L=5.3$ ) on September 22, 1999, 07:00-16:00 UT. The arrows indicate the time of SSC at 12:22 UT.

appearance, evident association with SSC). In the Section 4 we make an attempt to understand the origin of these two unusual phenomena. In conclusion (Section 5) we enumerate the main results of this study.

For the present study we have used mainly the pulsation magnetometer data from stations located in Finland. In addition, pulsation magnetometer data from Russian stations Borok and Mondy have been available. Table 1 presents the locations of these stations.

\section{Foreshock Effect: Pc 1 before SSC}

In accordance with our general idea on the searching for the terrestrial manifestations of the interplanetary fore- shocks (Kangas et al., 1998; Guglielmi et al., 2000), we have inspected the pulsation magnetometer records over the interval of several hours before SSC on September 22, 1999 at 12:22 UT. The foreshock part of the ULF waves appeared in the form of Pc 1 wave packet at 08:22 UT in the frequency band of $0.7-1.05 \mathrm{~Hz}$. It is seen at all observatories in Finland, but the signal is strongest at Ivalo and Sodankylä. The carrier frequency is $0.875 \mathrm{~Hz}$, the bandwidth $0.35 \mathrm{~Hz}$ and the duration is 6.5 minutes. Starting with this wave packet, the Pc 1 series develops during four hours till the time of SSC. Figure 1 presents the dynamic spectra of Pc 1 before sudden commencement at some selected stations.

We see that the mid-frequency of the initial Pc 1 series 


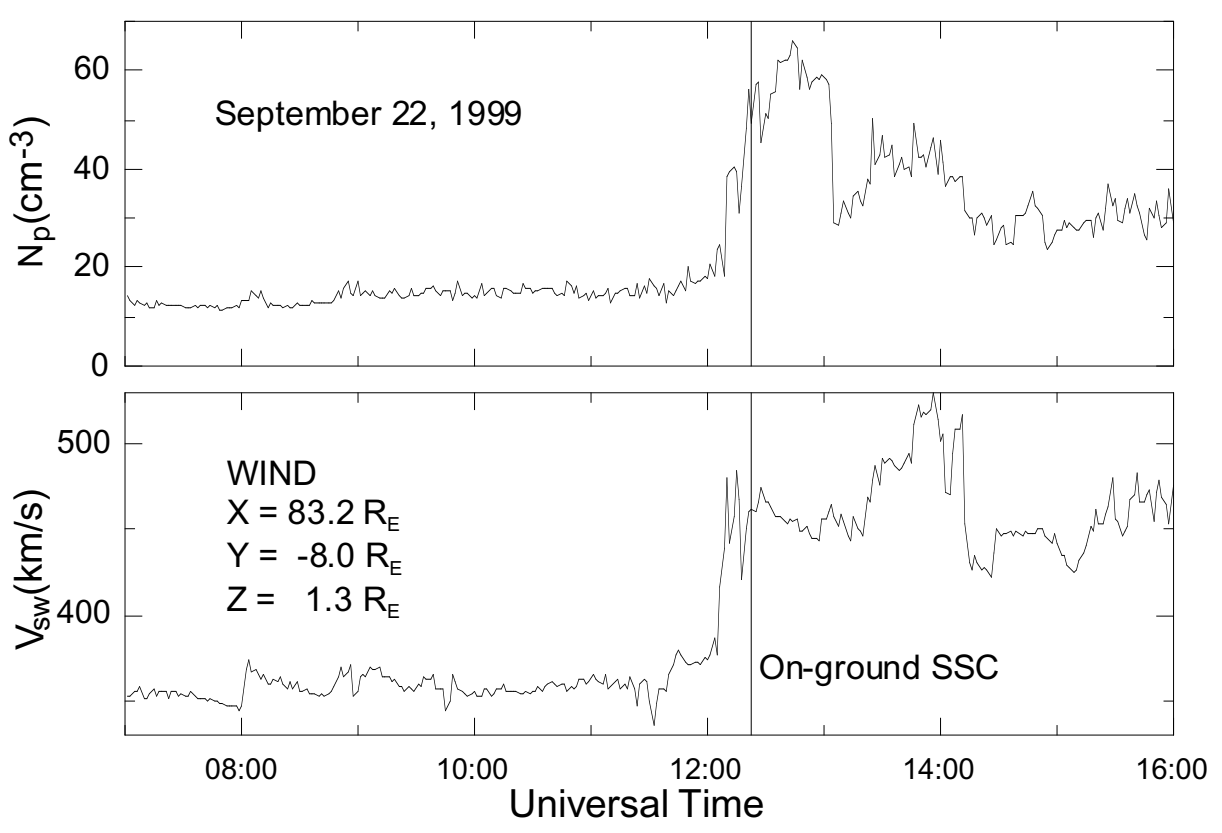

Fig. 2. Variations of solar wind velocity $V_{s w}$ and proton density $N_{p}$ measured on September 22,1999 , by WIND spacecraft approximately 70 earth radii from the bow shock upstream to the Sun. Vertical line shows the moment of SSC observation on the ground.

decreases slowly down to $0.82 \mathrm{~Hz}$. At 11:22 UT a new Pc 1 series begins at a lower frequency of $0.53 \mathrm{~Hz}$. The emission is modulated with alternating period of modulation: it is 24 minutes in the beginning, 14 minutes in the middle, and 8 minutes in the end of series. The mean modulation period is 16 minutes. At the same time a fine structure is noticeable in the dynamic spectra. The left-hand polarization dominates before SSC. It is seen especially in Kilpisjärvi where the amplitude of right-hand polarized signal is much less than that of the left-hand one. The $Y$-component is bigger than $X$-component. In dynamic spectra of the eastern observatories Borok and Mondy only weak, if any, traces of the pre-shock Pc 1 activity can be found.

In order to understand the nature of Pc 1 modulation we have analyzed conditions in the near-Earth solar wind before arrival of the interplanetary shock front associated with the SSC under consideration. In Fig. 2 we show WIND (Ogilvie et al., 1995) measurements of solar wind velocity $V_{s w}$ and proton density $N_{p}$. At 12:22 UT on September 22, 1999 the spacecraft was in the solar wind; its GSE coordinates were $[83.2,-8.0,1.3]$ Re. There are many interesting details in behavior of plasma parameters before SSC. According to interplanetary plasma variations shown in Fig. 2 a sharp increase in solar wind velocity is seen at 08:03 UT which is associated by a less pronounced increase in proton density. (This structure may be a result of nonlinear evolution of the upstream waves in the foreshock region.) Using the value of $370 \mathrm{~km} / \mathrm{s}$ as a typical transport velocity for the case under study we can estimate that the magnetosphere should be exposed by the foreshock region approximately at 08:25 UT. It coincides closely with the beginning of the pre-shock Pc 1 activity. Moreover, comparing the changes in the solar wind velocity with the modulation of Pc 1 intensity before the SSC we can notice a good correspondence between the two processes. For instance, variations of the solar wind velocity between 08:50 and 09:55 UT with the period of 10-20 minutes (Fig. 2) seem to trigger the 14-minute modulation of Pc 1 amplitude during 09:10 to 10:15 interval on the ground (Fig. 1). Somewhat later, 5-10 minute velocity oscillations between 11:00 and 12:00 UT (Fig. 2) are reflected in Pc 1 intensity variations of about 8-minute period at 11:20-12:20 UT on the ground (Fig. 1). In the Section 4 we will continue the discussion of these foreshock Pc 1 series and their connection with the solar wind variations.

\section{Post-Shock Effect: IPDP-Like Emission after SSC}

In Fig. 3 we present dynamic spectrum of the post-shock pulsations at Nurmijärvi and variations of the solar wind dynamic pressure with an expanded time scale as compared with Figs. 1 and 2. The moment of SSC on the ground (upper panel) is made coincident with the moment of the interplanetary shock transition through the satellite (bottom panel). In the upper panel we see that burst-like geomagnetic pulsations of rising frequency in the Pc 1 frequency range start soon after the sharp onset of the noise-like lowfrequency emission at 12:22 UT (this noise can also be seen at the bottom of sonagram in Fig. 1). The start of the first burst with a mean frequency of $1 \mathrm{~Hz}$ is delayed by $2 \mathrm{~min}$ with respect to the onset of the noise, during the following $36 \mathrm{~min}$ the mid-frequency of bursts grows up to $3.1 \mathrm{~Hz}$ at 12:57 UT. On the whole we can see five wide-band discrete patterns of rising tones. The frequency drift in these rising tones is of the order of $20-30 \mathrm{~Hz} / \mathrm{h}$. This frequency drift is more than one order bigger than that typical for the evening IPDP events (e.g., see Kangas et al., 1998). The typical value of the IPDP frequency drift is $1.3 \mathrm{~Hz} / \mathrm{h}$ in the lower frequencies, and $2.4 \mathrm{~Hz} / \mathrm{h}$ in the upper frequencies of the rising tones. A central frequency drifts with the rate of $d f / d t=1.8 \mathrm{~Hz} / \mathrm{h}$ (see, e.g., Pikkarainen et al., 1983). 

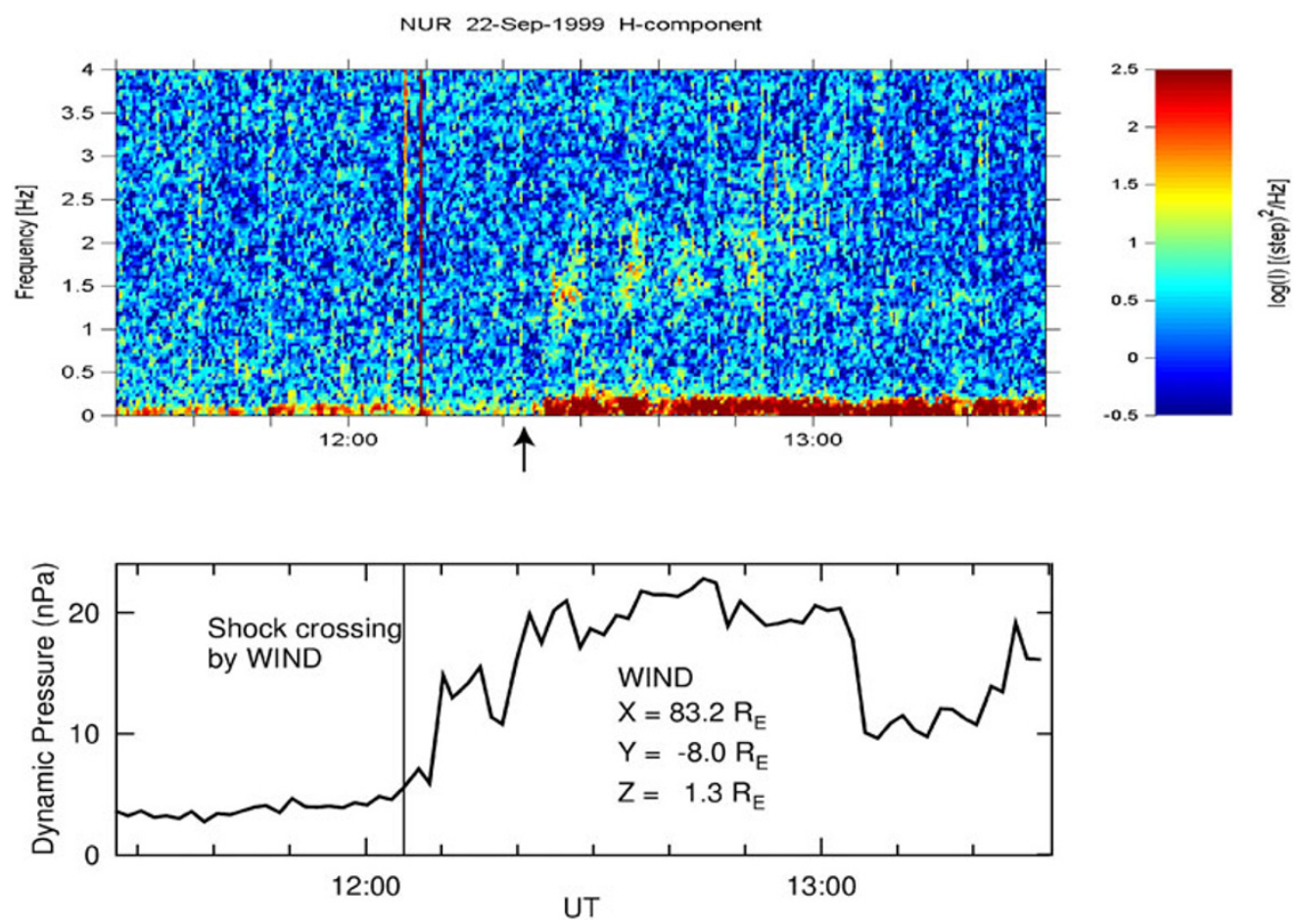

Fig. 3. Dynamic spectrum of post-SSC pulsations in Nurmijärvi $(L=3.4)$ (top panel) and variations of the solar wind dynamic pressure as calculated from WIND spacecraft measurements (bottom panel). The arrow shows the SSC moment on the ground. Vertical line at the bottom panel shows the moment of the interplanetary shock front transition through the satellite. Note that both moments are brought into coincidence to facilitate comparison of two processes.

Similar picture with some variations from place to place is observed at other stations, including Borok. However, the Mondy spectrogram shows no pulsation activity after SSC. The end frequency $f_{\text {end }}$ of the fifth burst decreases as the McIlwain parameter $L$ of the point of observation increases: $f_{\text {end }}=3.1 \mathrm{~Hz}$ in Nurmijärvi $(L=3.4)$ and $f_{\text {end }}=2.4 \mathrm{~Hz}$ in Kilpisjärvi ( $L=6.1)$. The left-hand polarization dominates in the aftershock event during the five emission bursts. In general, the $Y$-component is bigger than the $X$-component.

The discrete elements with rising tones after the SSC form an event which resembles the usual IPDP. However, there are two characteristics of the event which make it different from typical pulsations with diminishing period:

1. The event appears after SSC at 14:26 LT, that is in the day sector, whereas the usual IPDP waves grow after substorm onsets and they appear mainly in the evening and night sectors (see, e.g., the review by Kangas et al., 1998).

2. The most remarkable is the unusual high end frequency of the wave event. The value $f_{\text {end }}=3.1 \mathrm{~Hz}$ has never been observed in Nurmijärvi earlier. The average end frequency is $f_{\text {end }}=1 \mathrm{~Hz}$; up to date the highest value was $f_{\text {end }}=2 \mathrm{~Hz}$ (Pikkarainen, 1987; Kangas et al., 1998). In Kilpisjärvi the contrast is even more dramatic: on September 22, 1999 we observe $f_{\text {end }}=2.4$ $\mathrm{Hz}$, whereas the average value is $f_{\text {end }}=0.6 \mathrm{~Hz}$, and the highest value of $f_{\text {end }}=1 \mathrm{~Hz}$ up to date (Pikkarainen, 1987).

On the whole the observed Pc 1 event of September 22, 1999 , resembles very much an event of October 3, 1977, described in the paper by Kangas et al. (1986) (see figure 1 in that paper). Here we have the same intensification, spectrum widening, and a step-like increase in the frequency of the Pc 1 after SSC. It is pertinent to note in this connection that the nomenclature of post-SSC Pc 1 events varies somewhat in the literature. We prefer to use the term IPDP to designate the substorm associated evening pulsations of rising frequency. A designation for the post-SSC Pc 1 events of rising frequency may be introduced in the course of total revision of the ULF classification and nomenclature. We believe that such events form a special subclass of the ULF waves.

\section{Discussion}

The sonograms in Fig. 1 indicate without doubt that the Pc 1 before SSC relates genetically with the interplanetary shock front. We suppose that these Pc 1 waves are one of the magnetospheric manifestations of the interplanetary foreshock. It is known that "the foreshock is a region upstream of the shock that contains particles and waves associated with the shock" (Kivelson and Russell, 1995, p. 158). The waves and particles upstream from interplanetary blast shocks were detected in situ by Kennel et al. $(1982,1984)$ and Gosling et al. (1984). The geometry of a typical interplanetary shock front in the vicinity of the Earth's orbit (Hundhausen, 1972) predicts that the leading edge of the interplanetary foreshock comes into contact with the Earth's magnetosphere a few hours ahead of SSC. During these hours the periphery of magnetosphere and the high-latitude ionosphere are exposed by the charged particles accelerated by the shock and the wave turbulence upstream from the shock. 
Analyzing variations of the solar wind velocity and interplanetary plasma density before SSC shown in Fig. 2 we see that all these changes are evident in the modulation of the Pc 1 intensity. This indicates that the solar wind controls the ion cyclotron instability in the magnetosphere by means of penetration of pressure pulses from the solar wind into the magnetosphere. In other words, the interplanetary foreshock region interacting with the magnetosphere first launches the mechanism of ion cyclotron instability by a pressure pulse (08:20 UT), then modulates it by changing pressure inside the magnetosphere. In order to exclude another possible source of modulation, long-period Pc 3-4 pulsations, we have studied pulsation data from normal recordings at Sodankylä. Our conclusion was that Pc 5 pulsations were absent for the whole day of September 22, 1999. Pc 4 pulsations were recorded, but they were observed within the time interval 07:20-08:00 UT, that is before the beginning of the Pc 1 event. Therefore, we cannot say that in our case the modulation of Pc 1 is caused by the Pc 4-5 pulsations.

There are many unresolved problems pertaining to study the Pc 1 electromagnetic waves associated with the interplanetary foreshock regions. Specifically, it is not easy to explain why the mean frequency of the Pc 1 decreases gradually, as mentioned in the Section 2. In fact, a visible variation of the mid-frequency is within the accuracy of the analysis. If this variation takes really place, it may be explained, e.g., in terms of ponderomotive self-action of Pc 1 waves (Kangas et al., 1998). The essence of this mechanism is that the time-averaged ponderomotive forces caused by Pc 1 waves, when acting on charged particles, modify the dielectric properties of the medium, and thus also influence on the spectrum of Pc 1 waves. One of the possible ponderomotive effects is an increase of ion density along the path of wave propagation especially in the near-equatorial region. This leads to a decrease of Alfvén velocity $V_{A}$, and therefore to lowering of the resonant frequency $\omega \approx \Omega_{i}\left(V_{A} / v_{i}\right)$, where $\Omega_{i}$ is the gyrofrequency, and $v_{i}$ is the velocity of resonant ions. Yet another remarkable peculiarity of the dynamical spectra in Fig. 1 must be explained, namely, the appearance of a new Pc 1 series at frequency of $0.53 \mathrm{~Hz}$ immediately before SSC. The mechanism of this effect is open to speculation. We cannot do more than to mention two necessary conditions for the appearance of Pc 1 waves on the ground. The first condition is the existence of a field aligned magnetospheric waveguide; the second one requires that the free energy of ions within a particular waveguide is above the threshold. We propose that appearance of the $0.53 \mathrm{~Hz}$ band is the result of "activation" of an additional waveguide in the magnetosphere due to a pressure pulse in the solar wind.

One of the questions arising in relation to the pre-shock structure is whether such a structure with slightly increasing solar wind velocity and plasma density is typical for interplanetary shock waves. An additional example of similar structure for SSC on March 8, 1998, can be find in the paper by Guglielmi et al. (2000). We consider classification of the pre-shocks to be a subject of the future work. Another question is what type of foreshock causes the pre-shock Pc 1 with what mechanism and what type of foreshock does not. Our hypothesis is that the generation mechanism is related to the compression of the magnetosphere under the ponderomotive

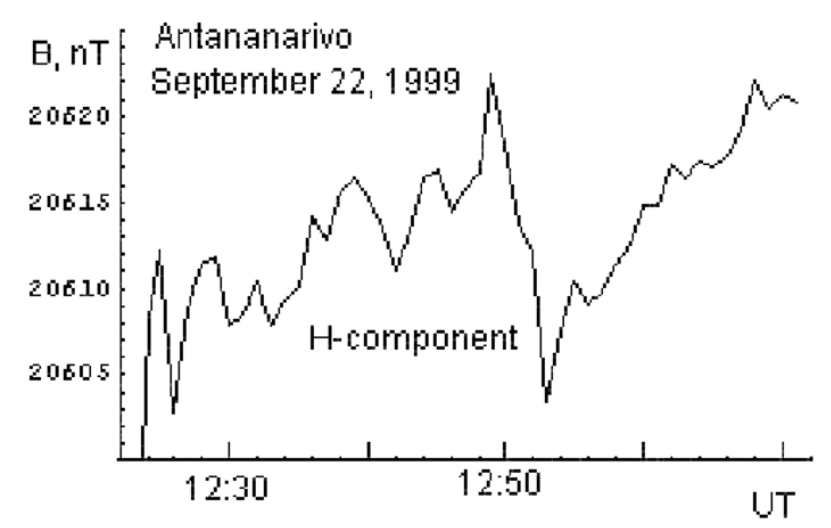

Fig. 4. Variations of $H$-component of geomagnetic field at the near-equatorial observatory Antananarivo during interaction of the interplanetary magnetic shock with the magnetosphere.

action of the plasma wave turbulence in the interplanetary foreshock region. However, the probability of excitation of Pc 1 depends not only on the intensity of the wave turbulence but also on the current state of the magnetosphere and ionosphere. The statistical study of this probability is also the subject of future publications.

Pc 1 wave emissions after the SSC of the present case reveal new details of the generation of Pc 1 waves due to the shock. We could consider these rising tones as a result of modification of Pc 1 by SSC as discussed e.g. by Kangas et al. (1986). It is worth to note that the preceding magnetic activity was low (Kp was equal 20,2 , and 3 - for three 3-hr intervals before SSC) in accordance with Kangas et al. (1986). But we cannot ignore the frequency increase in successive elements. On the other hand, Fig. 3 shows that variations of the solar wind dynamic pressure related to the interplanetary shock front have a modulation which can be associated with the emission bursts recorded on the ground. It seems that pulsations at this stage (Fig. 3, upper panel) reflect the fine structure of the shock having a form of dynamic pressure pulses which contract the magnetosphere by a series of powerful pushes. The manifestation of these pulses on the ground is seen also in the low-latitude magnetogram, see Fig. 4 where $H$-component variations are shown for Antananarivo observatory (geographic coordinates are latitude $=-18.9^{\circ}$, longitude $=47.6^{\circ}$ ).

So the overall frequency growth in successive structure patterns can be explained by compression of the magnetosphere in response to increasing dynamical pressure of the solar wind. The magnetosphere compression leads to an overall increase of the geomagnetic field, and also to the displacement of charged particles across the magnetic shells due to electric field drift (see e.g., Kangas et al., 1998). This enhances the anisotropy of resonant ions in the region of wave generation lowering the threshold of the ion-cyclotron instability and leads to the generation of higher frequencies as soon as the frequency of the increment maximum is proportional to the gyrofrequency of hot ions. Simultaneously the generation region itself expands which leads to frequency range widening in accordance with observations.

The slope of rising tones $(20-30 \mathrm{~Hz} / \mathrm{h})$ after SSC can 
be explained, e.g., by the frequency dispersion of Alfvén mode waves during their propagation to the ground under the assumption that the wave generation occurs at the inner magnetic shells with $L \sim 2-3$ or above the ionosphere at higher latitudes. An attempt to use the idea of wave dispersion of propagation velocity in presence of heavy ions has been made in paper by Lee and Kwok (1984) in order to explain frequency increase of IPDP events. It is of interest also to consider the effect of heavy ions on the properties of Pc 1 bursts after SI. As it is well known (Young et al., 1982), the content of oxygen and helium ions has its maximum in the years of high solar activity. In this respect it should be mentioned that Pc 1 bursts are also more common in the years of solar maximum in contrast to usual Pc 1 events (Kangas et al., 1986).

\section{Conclusion}

We report here a case study of the Pc 1 type of the ULF electromagnetic waves associated with the SSC at 12:22 UT on September 22, 1999. The main results are as follows:

1. We interpret the Pc 1 pulsations observed before the SSC as a further confirmation of the idea that these pulsations are a manifestation of the impact of the interplanetary foreshock (upstream) region on the geomagnetic field. In the paper by Guglielmi et al. (2000) it was shown statistically, here we have presented a case study with detailed description of the event in the frame of the S-RAMP September 1999 Space Weather Month campaign.

2. Observation of the post-shock wave emissions with rising tones revealed new details of their generation as a consequence of the shock interaction with the magnetosphere. Successive pressure pulses seem to intensify the wave generation. The slope of the frequency rise of successive bursts can be explained by the wave dispersion during wave propagation from the source region to the ground.

3. On the whole, due to previous papers devoted to SSC associated pulsations it seems that pre-shock and postshock emissions are two different, unrelated events. This paper shows that both events are not only connected by one cause, SSC, but have also one source, the magnetospheric compression.

In conclusion, this paper presents an evidence that the interaction of the Earth's magnetosphere with an interplanetary shock starts long before the direct contact of the shock front with the magnetopause. In the case of the event under study this interaction begins about $4 \mathrm{~h}$ before the contact, and it manifests itself in the excitation of Pc 1 magnetic pulsations with the amplitude modulated by the solar wind variations in the foreshock region.

Acknowledgments. We acknowledge K. W. Ogilvie (PI), A. J Lazarus and M. R. Aellig for WIND data on interplanetary plasma parameters taken from their Internet site. Two of us (A. G. and A. P.) would like to express the gratitude to Finnish Academy of Science and Letters and Academy of Finland for the financial support, and to the staff of Sodankylä Geophysical Observatory for the hospitality.

\section{References}

Anderson, B. J. and D. C. Hamilton, Electromagnetic ion cyclotron waves stimulated by modest magnetospheric compression, J. Geophys. Res. 98, 11369-11382, 1993

Baker, D. and H. Koskinen, S-RAMP September 1999 Space Weather Month and ISR World Days campaign update, SCOSTEP, Dec., 1999.

Gosling, J. T., S. J. Bame, W. C. Feldman, G. Paschmann, N. Sckopke, and C. T. Russell, Suprathermal ions upstream from interplanetary shocks, $J$. Geophys. Res., 89, 5409-5418, 1984.

Guglielmi, A. and O. Pokhotelov, Geoelectromagnetic Waves, IOP Publ Ltd, Bristol, 1996.

Guglielmi, A., J. Kangas, J. Kultima, and A. Potapov, An increase in Pc 1 wave activity prior to magnetic sudden impulses SI, J. Geophys. Res., 105, 25185-25189, 2000 .

Heacock, R. R. and V. P. Hessler, Pearl-type micropulsations associated with magnetic storm sudden commencements, J. Geophys. Res., 70, 1103-1111, 1965.

Hundhausen, A. J., Coronal Expansion and Solar Wind, Springer-Verlag, Heidelberg, New York, 1972.

Kangas, J., A. Aikio, and J. V. Olson, Multistation correlation spectra associated with sudden impulses, Planet. Space Sci., 34, 543-553, 1986.

Kangas, J., A. Aikio, and T. Pikkarainen, Radar electric field measurement during an IPDP plasma wave event, Planet. Space Sci., 36, 1103-1109, 1988

Kangas, J., A. Guglielmi, and O. Pokhotelov, Morphology and physics of short-period magnetic pulsations (A Review), Space Sci. Rev., 83, 435512, 1998.

Kennel, C. F., F. L. Scarf, F. V. Coroniti, E. J. Smith, and D. A. Gurnett, Nonlocal plasma turbulence associated with interplanetary shocks, $J$. Geophys. Res., 87, 17-34, 1982.

Kennel, C. F., F. L. Scarf, F. V. Coroniti, C. T. Russell, K.-P. Wenzel, C. R. Sunderson, P. Van Nes, W. C. Feldman, G. K. Parks, E. J. Smith, B. T. Tsurutani, F. S. Mozer, M. Temerin, R. R. Anderson, J. D. Scudder, and M. Scholer, Plasma and energetic particle structure upstream of a quasiparallel interplanetary shock, J. Geophys. Res., 89, 5419-5435, 1984.

Kivelson, M. G. and C. T. Russell (Eds.), Introduction to Space Physics, Cambridge, Univ. Press, 1995.

Kokubun, S. and T. Oguti, Hydromagnetic emissions associated with storm sudden commencements, Rep. Ionosph. Space Res. Japan, 22, 45-59, 1968.

Lee, L. C. and Y. C. Kwok, A mechanism for the IPDP pulsations, $J$ Geophys. Res., 89, 877-882, 1984.

Ogilvie, K. W., D. J. Chorney, R. J. Fitzenreiter, F. Hunsaker, J. Keller, J. Lobell, G. Miller, J. D. Scudder, E. C. Sittler, Jr., R. B. Torbert, D. Bodet, G. Needell, A. J. Lazarus, J. T. Steinberg, J. H. Tappan, A. Mavretic, and E. Gergin, SWE, a comprehensive plasma instrument for the Wind spacecraft, Space Sci. Rev., 71, 55-77, 1995.

Olson, J. V. and L. C. Lee, Pc 1 wave generation by sudden impulses, Planet. Space Sci., 31, 295-302, 1983.

Pikkarainen, T., Statistical results of IPDP pulsations recorded in Finland during 1975-1979, Geophysica, 23, 1-19, 1987.

Pikkarainen, T., J. Kangas, B. Kiselev, N. Maltseva, R. Rakhmatulin, and S. Solovjev, Type IPDP pulsations and the development of their sources, J. Geophys. Res., 88, 6204-6212, 1983.

Saito, T. and S. Matsushita, Geomagnetic pulsations associated with sudden commencements and sudden impulses, Planet. Space Sci., 15, 573-587, 1967.

Tepley, L. R. and R. C. Wentworth, Hydromagnetic emissions, X-ray bursts, and electron bunches. 1. Experimental results, J. Geophys. Res., 67, 3317-3333, 1962

Troitskaya, V. A., Pulsations of the Earth's electromagnetic field with periods of 1 to $15 \mathrm{~s}$ and their connection with phenomena in high atmosphere, J. Geophys. Res., 66, 5-18, 1961.

Troitskaya, V. A., Rapid variations of the electromagnetic field of the Earth, in Research in Geophysics, edited by H. Odishaw, M.I.T. Press, Cambridge, Mass., Vol. 1, pp. 485-532, 1964.

Troitskaya, V. A. and A. V. Guglielmi, Geomagnetic micropulsations and diagnostics of the magnetosphere, Space Sci. Rev., 7, 689-768, 1967.

Young, D. T., H. Balsiger, and J. Geiss, Correlations of magnetospheric ion composition with geomagnetic and solar activity, J. Geophys. Res., 87, 9077-9096, 1982

J. Kangas, J. Kultima (e-mail: uula@sgo.fi), A. Guglielmi, A. Potapov, and K. Hayashi 